\title{
Simulación técnica económica de dos sistemas de construcción de techumbre en el sureste de México
}

\section{Technical and economical simulation of two roofing construction systems in southeast M exico}

\author{
Julio R. Baeza Pereyra*1, Sergio 0. Álvarez Romero*
}

* Universidad Autónoma de Yucatán, M ÉXICO

Resumen

Fecha de recepción: 02/ 04/2009 Fecha de aceptación: 30/ 06/ 2009 PAG. $167-180$

El uso de prefabricados en la construcción aparentemente no es una alternativa económica si se le compara con métodos constructivos que hacen uso de mano de obra de manera intensiva en países en vías de desarrollo. En este trabajo se presentan un estudio comparativo de dos alternativas de construcción de techumbre en construcción de vivienda masiva en el sureste de México. El sistema tradicional de construcción, que usa mano de obra intensiva y el sistema de losas prefabricadas L-18 fueron modeladas utilizando el sistema de simulaciones computarizadas EzStrobe. Se realizó un estudio técnicoeconómico entre ambos sistemas, comparando la relación entre sus insumos, los costos directos e indirectos de administración. En lo que se refiere al costo directo se observó que el método prefabricado es mayor que el método tradicional, sin embargo, el sistema prefabricado es capaz de ajustar y adaptar su precio de venta en la planta, en función de un volumen determinado de obra.

Palabras Clave: Simulación computarizada, costo directo, costos indirectos, análisis comparativo

Abstract

The use of prefabrication in construction apparently is not a economic alternative if compared with more labor intensive construction methods in developing countries. This work presents a comparison study between two choices of roofing systems for massively housing construction in southeast Mexico. The traditional labor intensive roofing construction and the prefabricated roofing construction system coded L-18 were modeled using the computer program EzStrobe simulation software. A technical and economical analysis was performed on both construction methods, comparing the relationship between resources, direct cost, and indirect administrative cost. In reference to the direct cost, the prefabricated construction method cost was higher than the traditional method; however, the prefabricated system is capable of adjusting its selling price in the production factory according to a given construction volume.

Keywords: Computer-assisted simulation, direct cost, indirect cost, comparative analysis

\section{Introducción}

El sistema actual de fabricación de losas para vivienda en el contexto del sureste de México, ha sido desde años atrás el concepto de obra con el mayor porcentaje del costo dentro del costo directo total de una vivienda de acuerdo con el estudio realizado por Silvia Campos (Campos, 1985). En lo que se refiere al tiempo de ejecución y la calidad de este concepto de obra se han podido identificar algunos defectos que se presentan debido a que en la mayoría de las veces no se cuenta con una supervisión adecuada de los recursos materiales suministrados por el proveedor,

\section{Introduction}

The curren roofing construction system for housing in southeast Mexico, has been the same since long ago, the labor item that represents the highest percentage of the housing total direct cost, according to the study developed by Silvia Campos (Campos, 1985). As far as the execution time and the quality of this labor item are concerned, some deficiencies have been found, mainly derived from the lack of a proper supervision on the material delivered by the supplier,

${ }^{1}$ Autor de correspondencia / Corresponding author:
E-mail: bpereyra@uady.mx 
no se tiene un sistema de control administrativo y mucho menos se toman las precauciones constructivas para su fabricación (Gutiérrez, 1995). Aunado a lo anterior se derivan otros problemas como la cantidad de desperdicios de la materia prima, el esfuerzo humano de los trabajadores, las perdidas de productividad por los tiempos de espera del fraguado de la losa y el empleo de cimbra de apuntalamiento (Pinzón, 1990) (Figura 1). the lack of a managing control system, and construction precautions standards are not met (Gutiérrez, 1995). Besides, there are some other problems such as the waste amount of raw material, labor hand effort and the lack of productivity during waiting time while roof hardens and the centering support is employed. (Pinzón, 1990) (Figure 1).

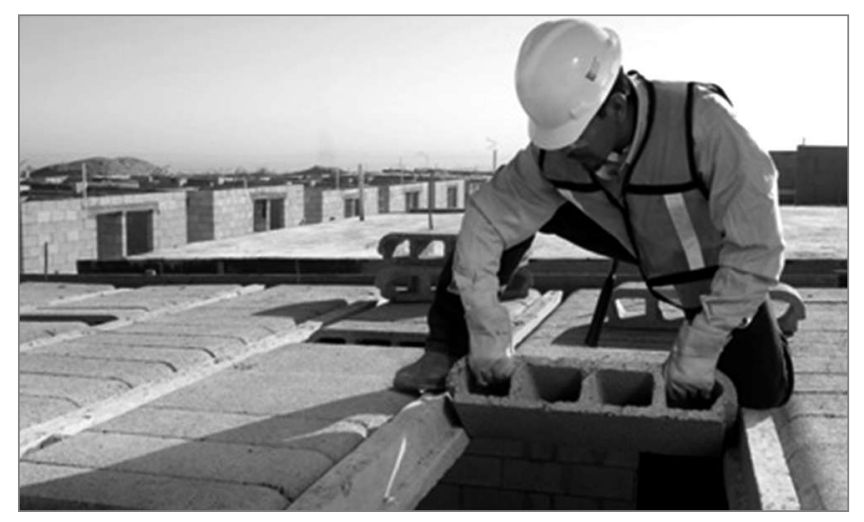

Figura 1. Sistema losa nervada de vigueta y bovedilla tradicional Figure 1. Traditional system of ceiling hoist and vault in a ribbed slab

Por otro parte se sigue demostrando actualmente en los países desarrollados, que la tendencia de los procesos constructivos gira en torno a la prefabricación como una medida de un desarrollo más tecnológico, industrializado y sustentable para la construcción, ya que el empleo de esta técnica constructiva representa grandes ahorros, ventajas y beneficios tanto para las empresas constructoras, como para los clientes, la sociedad y el medio ambiente (Yee, 2001).
On the other hand developed countries have proved so far, that the trends for constructive processes are centered in precasting, since it is a more industrialized and supportable construction method; thus representing great savings, profits and benefits for construction companies, as well as for clients, society and the environment (Yee, 2001).

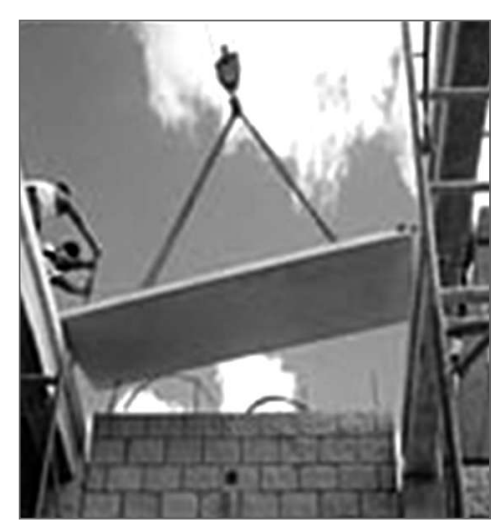

Figura 2. Losa prefabricada L-18 (proceso de montaje) Figure 2. Precast roofing, code L-18 (assembly process) 
En la Facultad de Ingeniería de la U niversidad Autónoma de Yucatán se han llevado a cabo esfuerzos por crear esquemas y modelos para realizar una comparación cuantitativa entre el método tradicional de construcción de techumbres para proyectos de vivienda masiva tradicional (Baeza, 2008) y un sistema de construcción de techumbres prefabricadas, designada como L-18 (Sosa et al., 2007). En ambos trabajos se recolectó información acerca de la productividad de la mano de obra y de los equipos involucrados en los procesos. Así mismo se realizó un estudio de flujo de trabajo para determinar el esquema que ambos procedimientos seguían en su proceso constructivo. Con dicha información se programaron sendos modelos de simulación computarizada para cada modelo individual.

Los referidos modelos fueron ejecutados en una computadora personal, utilizando el sistema EzStrobe (Martínez, 1998). Sosa et. al encontraron que el sistema L-18 se tardaría 299.18 minutos (cerca de 5 hrs.) en cubrir un lote de 4 losas. Por otro lado, Baeza encontró que usando el modelo de losa tradicional de la región, el proceso de cubrir cuatro losas tardaría 842 minutos (lo que equivale a $14 \mathrm{hrs}$. de una jornada larga 01.75 jornadas de $8 \mathrm{hrs}$.).

Lo que es obvio de notar es la gran ventaja que el método L-18 es más económico en cuestión de tiempo, por encima del método tradicional. Sin embargo, esta economía en tiempo no se traduce en una economía en costo, sobre todo en la región sureste de México, donde es mucho más barato el uso de mano de obra. En contraposición, el uso de maquinaria tiene un costo de inversión inicial en maquinaria rentada mayor que el solo uso de mano de obra (Sosa, 2005)

Por lo anteriormente descrito, para comparar a estos dos sistemas de construcción, se hace necesario conocer cual es su comportamiento a nivel de construcción masiva, partiendo de la hipótesis de que si el volumen de construcción de viviendas es grande, deberá de existir un punto de equilibrio entre ambos procesos constructivos, donde uno de ellos será más económico que el otro en términos monetarios.

\section{M etodología}

Para comparar estos dos sistemas constructivos se llevaron realizaron los siguientes pasos:

1. Se llevó a cabo un ajuste de los modelos de
The Engineering Department at U niversidad Autónoma de Yucatán has made some efforts to create new diagrams and models to carry out a quantitative comparison between the traditional roofing construction method for massive traditional housing (Baeza, 2008) and the precast roofing method, coded L-18 (Sosa et. al, 2007). Both studies collected information about labor hand productivity and the equipment involved in the construction. A workflow study was made in order to determine the method that both processes followed. Such information was used to create simulation software to each individual model.

The referred models were run in a PC by using the EzStrobe simulation software (Martínez, 1998). Sosa et al. found that the system coded L-18 would take 299.18 minutes (app. 5 hours) to cover a lot of 4 roofs. On the other hand, Baeza found that using the same traditional roofing model in the region, the process would take 842 minutes (which is equivalent to $14 \mathrm{hrs}$ in a long day's work or 1.75 eight hours in a day`s work).

It is obvious that method L-18 represents a great advantage as far as execution time is concerned, since it is cheaper than the traditional method. However, this saving is not translated into an effective cost saving, especially in the southeast M exico region, where labor hand is a unexpensive. The use of machinery has an initial investment cost higher for rented machinery than the use of labor hand only. (Sosa, 2005)

As described above, in order to compare these two systems, it is required to learn about the behavior at a massively construction level, beginning with an unproved theory: if the volume of massively housing construction is large enough, there would be a balance point between both constructive processes, where one of them will be more economic than the other in financial terms.

\section{Methodology}

In order to compare these two constructive systems the following steps were taken.

1. An adjustment to Baeza`s and Sosa's simulation 
simulación de Baeza y de Sosa, para adecuarlos al contexto de construcción masiva ${ }^{1}$

2. Para obtener los costos de los recursos se utilizó la tesis de Sosa (Sosa, 2005), en la cual se tiene recopilada la información, tanto de costos directos, como de indirectos de operación para ambos sistemas constructivos.

3. De dicho trabajo se recopiló los esquemas de trabajo y recursos necesarios para la simulación de ambos sistemas constructivos

4. Se simularon los dos métodos constructivos, programando los modelos producidos en el primer paso, para obtener la duración en jornales de construcción de losas, para ambos procesos constructivos.

5. Se realizó un análisis de precios unitarios por losa construida por ambos procesos constructivos. También se realizó un análisis de relación de Costo Indirecto por Costo Directo de Producción en ambos casos.

\section{Resultados}

\subsection{Modelos de Simulación}

Inicialmente se hicieron modificaciones para el sistema de Sosa et al., 2007 con el propósito de adecuarlo al contexto de simulación de construcción masiva. Este modelo modificado se presenta en la Figura 3.

Entre las mejoras que tiene el modelo de la

Figura 3 sobre el presentado por Sosa, Baeza y Arcudia (2007), es que se le han añadido un punto donde se puede modificar información ya sea del número de casas, del número de maquinas, obreros, así como demoras que se puedan sufrir por cuestiones de diversa índole. La Tabla 1 detalla los componentes de dicho modelo. models were made, so as to make them fit into a massively construction context ${ }^{1}$.

2. In order to obtain the resources costs, Sosa's thesis was employed (Sosa, 2005), which contains the information about direct and non-direct operation costs for each constructive systems.

3. From such study the work diagrams and necessary resources where obtained for the simulation of both constructive systems.

4. The two constructive methods were simulated, first programming the models to obtain the roofing constructions execution time expressed in day's work, for both constructive processes.

5.An analysis on unit prices per roofing constructed was made for both constructive processes. Another analysis was also made related to $\mathrm{N}$ on-direct Cost per Direct Production Cost in both cases.

\section{Results}

\subsection{Simulation Models}

Initially some modifications were made for the system Sosa et al., 2007, in order to make it fit into the massively construction simulation context. This modified model is shown in Figure 3.

Some improvements were made to the model presented by Soza, Baeza and Arcudia (2007); thus Figure 3 includes an entry point where information can be modified, such as number of houses, number of equipments, number of workers, as well as delays due to different kinds of nature.

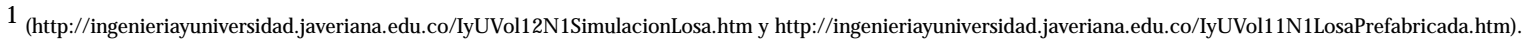




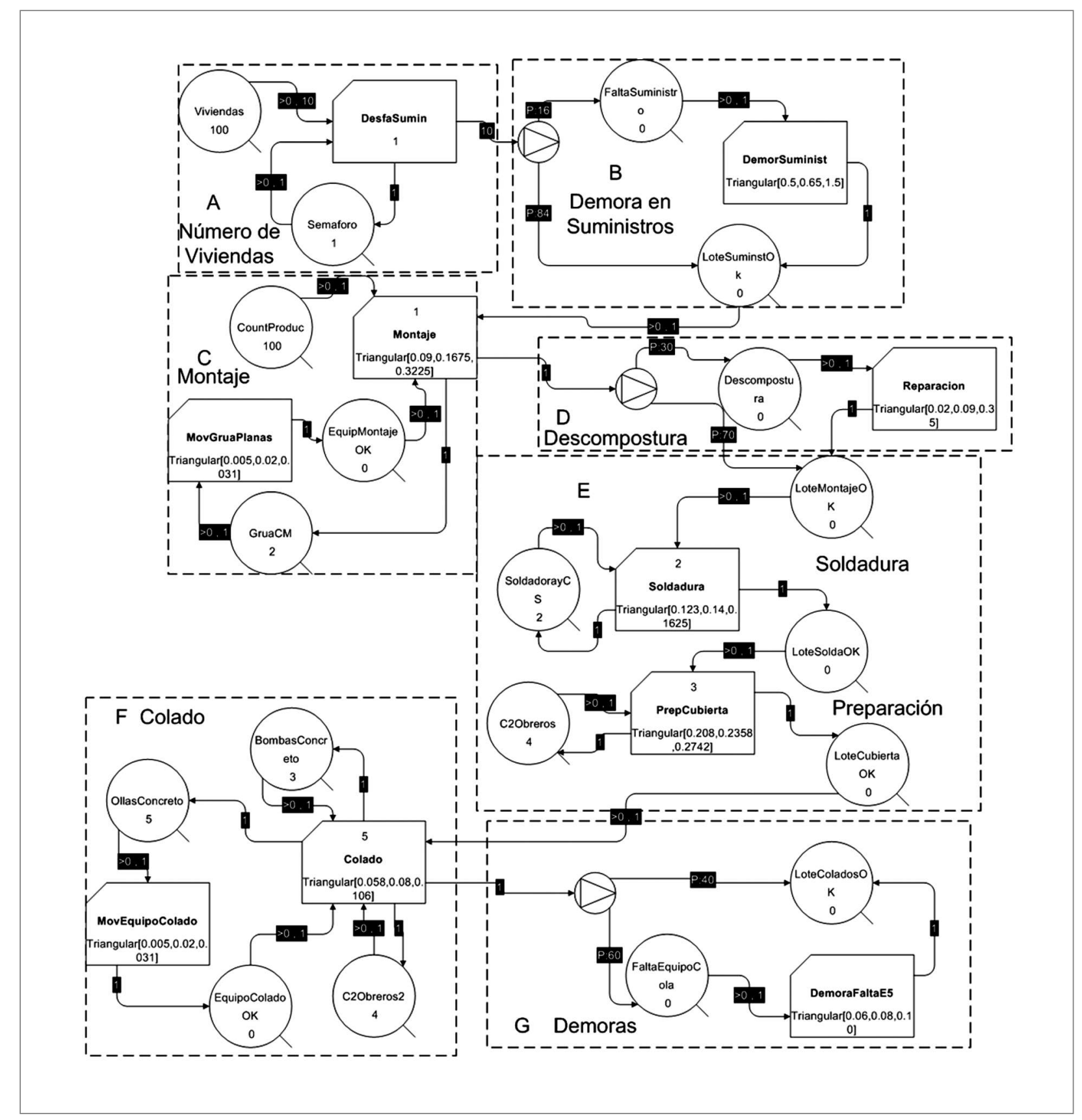

Figura 3. Modelo de construcción masiva de losas de viviendas, utilizando el sistema de prefabricado L-18 Figure 3. Massively Housing Roofing Construction Model using the precast system L-18 
Tabla 1. Detalles del modelo en la Figura 3, sistema L-18 masivo Table 1. D etails of model in figure 3 , massive system L-18

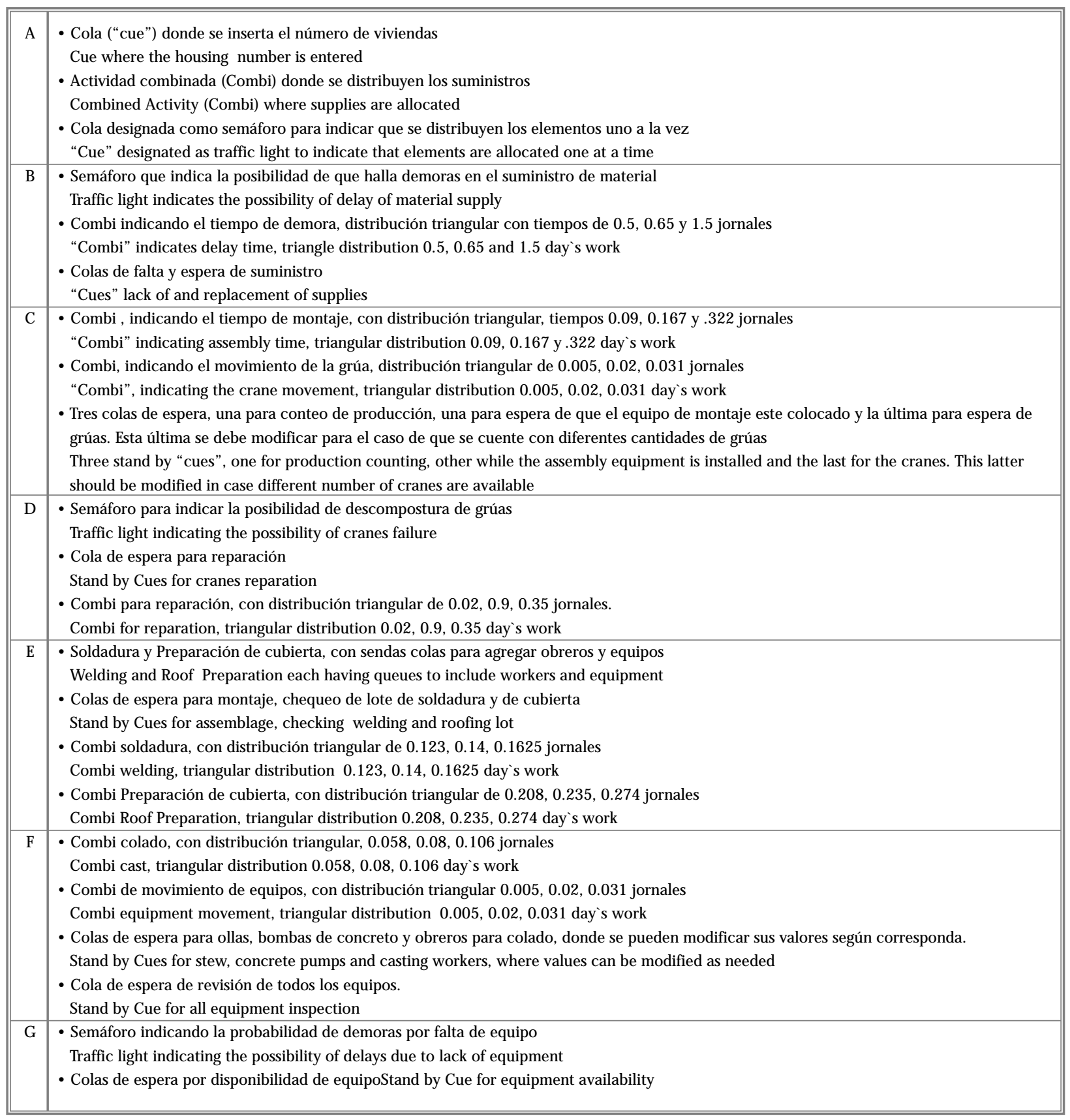

Para el método tradicional, el modelo original del que se partió se encuentra explicado en Baeza (2008). Dicho modelo se modificó de manera similar al de Sosa et al., 2007, añadiéndole nodos para incluir obreros, mezcladoras de hormigón, demoras por falta de insumos y revisiones. El modelo resultante se muestra en la Figura 4. La Tabla 2 muestra la información del modelo en detalle.
The original model used for the traditional method is explained in Baeza (2008). Such model was modified as done with Sosa et. al., 2007 model, including nodes to register number of workers, concrete mixers, delays due to lack of raw material and inspections. The resulting model is shown in Figure 4. Table 2 shows detailed information of the model. 


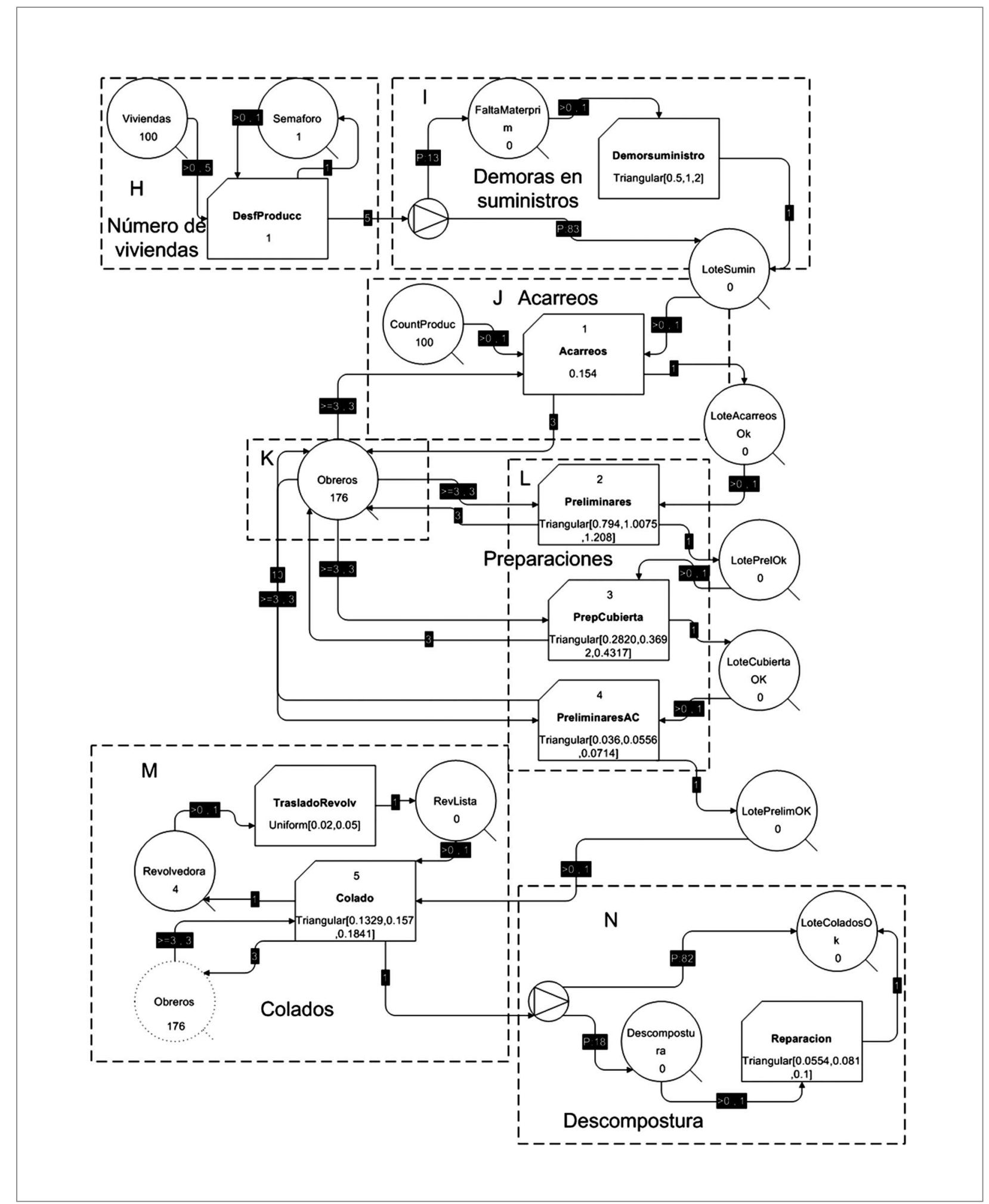

Figura 4. Modelo de construcción masiva de losas de viviendas, utilizando el sistema tradicional de vigueta y bovedilla Figura 4. Massively Roofing Housing Construction Model, using the traditional ceiling joist and vault system 
Tabla 2. Detalles del modelo de la figura 4, método tradicional masivo

Table 2. Details of model shown in Figure 4, massively traditional method

\begin{tabular}{|c|c|}
\hline $\mathrm{H}$ & $\begin{array}{l}\text { - Nodo de cola para preparar las losas que hay que fabricar. Este dato es una variable de entrada } \\
\text { Cue anode to prepare the roofs to be constructed. This data is an entry index } \\
\text { - Actividad combinada (Combi) donde se distribuyen los suministros/Combined activity (Combi) where supplies are allocated } \\
\text { - Cola designada como semáforo para indicar que se distribuyen los elementos uno a la vez } \\
\text { Cue designated as traffic light to indicate that elements are allocated one at a time }\end{array}$ \\
\hline 1 & $\begin{array}{l}\text { - Semáforo para distribuir las probabilidades de demora de suministros/Traffic light to allocate the possibility of supplies delays } \\
\text { - Cola de espera por falta de material/Stand by Cue due to lack of material } \\
\text { - Combi para cuantificar la demora de materiales, con distribución triangular de } 0.5,1,2 \text { jornales } \\
\text { Combi to estimate material delay, triangular distribution } 0.5,1,2 \text { day`s work }\end{array}$ \\
\hline$J$ & $\begin{array}{l}\text { - Cola de espera por producción de unidades/Stand by Cue for units production } \\
\text { - Cola de espera por suministros/Stand by Cue for material supply } \\
\text { - Combi de acarreos, con una duración fija de } 0.154 \text { jornales/Transportation Combi, fixed duration } 0.154 \text { day`s work } \\
\text { - Cola de espera por acarreos/Stand by Cue for transportation }\end{array}$ \\
\hline K & $\begin{array}{l}\text { - Cola de espera por obreros. La cantidad de obreros es una variable dada por el usuario } \\
\text { Stand by Cue for workers. Workers number is provided by the user }\end{array}$ \\
\hline $\mathrm{L}$ & $\begin{array}{l}\text { - Combi para las actividades preliminares, con distribución triangular de } 0.794,1.005,1.208 \text { jornales } \\
\text { Combi for preliminary activities, triangular distribution } 0.794,1.005,1.208 \text { day`s work } \\
\text { - Cola de espera por término de preliminares/Stand by Cue for preliminary activities completion } \\
\text { - Combi para la preparación de la cubierta, con distribución triangular de } 0.282,0.369,0.4317 \text { jornales } \\
\text { Combi for roof preparation, triangular distribution } 0.282,0.369,0.4317 \text { day`s work } \\
\text { - Cola de espera para la preparación de la cubierta/Stand by Cue for roof preparation } \\
\text { - Combi para las actividades antes del colado, con distribución triangular de } 0.036,0.555,0.071 \text { jornales } \\
\text { Combi for activities previous casting, triangular distribution } 0.036,0.555,0.071 \text { day`s work } \\
\text { - Cola de espera por preliminares antes del colado/Stand by Cue for preliminary activities before casting }\end{array}$ \\
\hline$M$ & $\begin{array}{l}\text { - Combi colado de hormigón, con una distribución triangular de } 0.133,0.157,0.184 \text { jornales } \\
\text { Combi concrete cast, triangular distribution } 0.133,0.157,0.184 \text { day`s work }\end{array}$ \\
\hline $\mathrm{N}$ & $\begin{array}{l}\text { - Cola de espera por obreros para la losa que se está fabricando, datos tomados de la sección K. } \\
\text { - Stand by Cue for workers constructing the roof, data taken from section } \mathrm{K} \\
\text { - Cola de espera por Revolvedora. Este dato se puede modificar para incluir el numero de revolvedoras necesarias para la fabricación } \\
\text { Stand by Cue for M Mxer. Data can be modified to include number of mixers required for the fabrication } \\
\text { - Combi para traslados de revolvedora, con una distribución uniforme de } 0.02 \text { a } 0.05 \text { jornales } \\
\text { Combi for mixer transportation, fixed distribution } 0.02 \text { a } 0.05 \text { day`s work } \\
\text { - Cola de espera por revisión de lista de actividades/Stand by Cue Cola for check list activities } \\
\text { - Semáforo que indica la posibilidad de descompostura de revolvedora./Traffic light indicates the possibility of mixer failure } \\
\text { - Cola de espera para reparación de revolvedora/Stand by Cue for mixer reparation } \\
\text { - Combi para la reparación de revolvedora, con una distribución de } 0.055,0.081,0.1 \text { jornales } \\
\text { Combi for mixer reparation, distribution } 0.055,0.081,0.1 \text { day`s work } \\
\text { - Cola de recepción de losas fabricadas/Stand by Cue for finished roofing reception }\end{array}$ \\
\hline
\end{tabular}

\subsection{Costos para la simulación}

Asumiendo un lote tipo de losa de $45 \mathrm{~m}^{2}$ por unidad habitacional, el costo directo promedio que se usó para este trabajo fueron tomados de la tesis de maestría de Sosa (Sosa, 2005). Los precios están en pesos mexicanos de 2005

\subsection{Simulation costs}

Considering a given $45 \mathrm{~m}^{2}$ roofing lot per housing unit, the average direct cost used for this work was taken from Sosa's mastery thesis. (Sosa, 2005). Prices are expressed in mexican pesos of 2005.

Tabla 3. Costos estadísticos para la simulación de losa tradicional

Table 3. Statistical Costs for traditional roofing system simulation

\begin{tabular}{||l|c|}
\hline \hline Etapas o partidas/Steps or Items & Costo promedio/Average Cost \\
\hline Acarreos/Transportation & $\$ 90.00$ \\
\hline Preliminares/Precasting & $\$ 4,833.99$ \\
\hline Preparación de cubierta/Roofing preparations & $\$ 550.17$ \\
\hline Preliminares antes del colado/Cast before precasting & $\$ 1,571.36$ \\
\hline Colocación del Hormigón/Concrete assemblage & $\$ 1,390.36$ \\
\hline Costo-Losa/Cost per Roofing System & $\$ 8,435.88$ \\
\hline
\end{tabular}


Tabla 4. Costos estadísticos para la simulación de losa prefabricada L-18

\begin{tabular}{||l|c||}
\hline Etapas o partidas/Stages or Items & Costo promedio/Average Cost \\
\hline Costo de venta de piezas L-18/Sales Price for L-18 items & $\$ 8,963.10$ \\
\hline Rigidización/W edge In & $\$ 81.00$ \\
\hline Costo de Mano de O bra para preparar la cubierta/Labor hand, roof preparations & $\$ 281.25$ \\
\hline Colocación del Hormigón/Concrete assemblage & $\$ 2,250.00$ \\
\hline Costo-Losa/Cost per Roofing System & $\$ 11,575.35$ \\
\hline
\end{tabular}

El costo indirecto proporcionado por una constructora local de tamaño mediano que construye un número significativo de viviendas al año, fueron índices unitarios que resultaron de realizar las operaciones aritméticas que se encuentran en la tesis de Sosa (Sosa, 2005). En relación al costo indirecto involucra los rubros de indirecto más importantes para una constructora que varían en función del tiempo (sueldos de oficina y campo, prestaciones y gastos de operación). El valor del costo indirecto de la empresa investigada por Sosa es de $\$ 507.27$

\subsection{Esquema de trabajo}

Se llevó a cabo una entrevista con un residente de obra que explicó cual sería la logística para llevar a cabo diversos volúmenes de obra. De dicha entrevista se tomaron los datos para el estudio de recursos necesarios para la simulación. Las tablas 5 y 6 muestran los esquemas de recursos que se utilizaron para la simulación de ambos sistemas de construcción.
A middle size local construction company, that builds an important number of housing units per year, provided the non-direct costs which were processed according to Sosa's thesis calculation (Sosa, 2005); thus obtaining the unit index. Regarding the non-direct costs involved, the most important non-direct items for a construction company also vary as far as time is concerned (office and field salaries, services payment and operational costs). The amount of non-direct cost provided by the company researched by Sosa is $\$ 507.27$

\subsection{Work Plan}

In an interview the building supervisor explained which would be the supply chain methods required to develop several construction volumes. Valuable data obtained from such interview were used on the resources study required for the simulation. Tables 5 and 6 show the resources diagrams used for the simulation on both construction systems.

Tabla 5. Análisis de productividad losa tradicional

\begin{tabular}{|c|c|c|c|}
\hline \multirow[b]{2}{*}{ No. LOSAS/RO OFING NUMBER } & \multicolumn{2}{|c|}{ RECURSOS UTILIZADOS/USED RESO URCES } & DUR./EXECUTION TIME \\
\hline & PERSO NAL/W O RKERS & REVOL./MIXER & SIMULACIÓN (DÍAS)/SIM U LATION (DAYS) \\
\hline 1 & 2 & 1 & 2.73 \\
\hline 5 & 10 & 1 & 4.45 \\
\hline 10 & 20 & 1 & 4.89 \\
\hline 15 & 30 & 1 & 5.47 \\
\hline 20 & 40 & 1 & 6.4 \\
\hline 25 & 50 & 1 & 8.24 \\
\hline 30 & 60 & 2 & 8.1 \\
\hline 35 & 70 & 2 & 10.44 \\
\hline 40 & 80 & 2 & 10.06 \\
\hline 45 & 90 & 2 & 11.11 \\
\hline 50 & 100 & 2 & 12.09 \\
\hline 55 & 110 & 2 & 13.18 \\
\hline 60 & 120 & 2 & 14.25 \\
\hline 65 & 130 & 3 & 14.95 \\
\hline 70 & 140 & 3 & 16.31 \\
\hline 75 & 150 & 3 & 16.94 \\
\hline 80 & 160 & 3 & 17.92 \\
\hline 85 & 170 & 4 & 18.87 \\
\hline 90 & 180 & 4 & 19.85 \\
\hline 95 & 190 & 4 & 20.91 \\
\hline 100 & 200 & 4 & 21.87 \\
\hline
\end{tabular}


Julio R. Baeza Pereyra, Sergio O. Álvarez Romero

Tabla 6. Análisis de productividad Iosa prefabricada L-18

Tabla 6 . Productivity analysis for the precast L-18 roofing system

\begin{tabular}{|c|c|c|c|c|c|c|c|c|}
\hline \multicolumn{8}{|c|}{ RECURSOS UTILIZADOS/U SED RESO U RCES } & \multirow[b]{2}{*}{$\begin{array}{l}\text { DUR. } \\
\text { TIME }\end{array}$} \\
\hline & $\begin{array}{l}\text { PLANAS } \\
\text { CRANE }\end{array}$ & $\begin{array}{l}\text { MONTAJE } \\
\text { ASSAMBL. }\end{array}$ & $\begin{array}{l}\text { SO LDADURA } \\
\text { WELDING }\end{array}$ & $\begin{array}{l}\text { PREP.LOSA } \\
\text { RO OF MIX. }\end{array}$ & & $\begin{array}{l}\text { COLADO } \\
\text { CAST. }\end{array}$ & & \\
\hline $\begin{array}{c}\text { N.LOSAS } \\
\text { ROOFING NR. }\end{array}$ & $\begin{array}{l}\text { REQ UE. } \\
\text { REQ. }\end{array}$ & $\begin{array}{c}\text { GRUA+M.O } \\
\text { CRANE+LABOR H. }\end{array}$ & $\begin{array}{c}\text { MAQ.+MO } \\
\text { EQ UIPMENT + LABOR H. }\end{array}$ & $\begin{array}{c}\text { CUAD.M.O } \\
\text { CREW LABOR H. }\end{array}$ & $\begin{array}{l}\text { OLLA } \\
\text { STEW }\end{array}$ & $\begin{array}{l}\text { BOMBA } \\
\text { PUMP }\end{array}$ & $\begin{array}{l}\text { C.M.O } \\
\text { C.L.H. }\end{array}$ & $\begin{array}{l}\text { SIMULACIÓN.(DÍAS) } \\
\text { SIMULATION (DAYS) }\end{array}$ \\
\hline 1 & 1 & 1 & 1 & 1 & 1 & 1 & 1 & 1.8 \\
\hline 5 & 1 & 1 & 1 & 1 & 1 & 1 & 1 & 2.84 \\
\hline 10 & 1 & 1 & 1 & 2 & 2 & 1 & 2 & 3.83 \\
\hline 15 & 1 & 1 & 1 & 2 & 2 & 1 & 2 & 4.67 \\
\hline 20 & 1 & 1 & 1 & 2 & 2 & 2 & 2 & 5.84 \\
\hline 25 & 2 & 1 & 1 & 2 & 2 & 1 & 2 & 6.91 \\
\hline 30 & 2 & 1 & 1 & 2 & 2 & 1 & 2 & 8.01 \\
\hline 35 & 2 & 1 & 1 & 2 & 2 & 1 & 2 & 9.24 \\
\hline 40 & 2 & 1 & 1 & 2 & 2 & 1 & 2 & 10.44 \\
\hline 45 & 3 & 2 & 2 & 2 & 3 & 1 & 2 & 8.85 \\
\hline 50 & 3 & 2 & 2 & 2 & 3 & 1 & 2 & 9.72 \\
\hline 55 & 3 & 2 & 2 & 2 & 3 & 1 & 2 & 10.8 \\
\hline 60 & 4 & 2 & 2 & 3 & 4 & 2 & 3 & 11.2 \\
\hline 65 & 4 & 2 & 2 & 3 & 4 & 2 & 3 & 9.7 \\
\hline 70 & 4 & 2 & 2 & 3 & 4 & 2 & 3 & 10.25 \\
\hline 75 & 4 & 2 & 2 & 3 & 4 & 2 & 3 & 10.96 \\
\hline 80 & 4 & 2 & 2 & 3 & 4 & 2 & 3 & 11.62 \\
\hline 85 & 5 & 2 & 2 & 4 & 5 & 3 & 4 & 10.46 \\
\hline 90 & 5 & 2 & 2 & 4 & 5 & 3 & 4 & 11.71 \\
\hline 95 & 5 & 2 & 2 & 4 & 5 & 3 & 4 & 12.55 \\
\hline 100 & 5 & 2 & 2 & 4 & 5 & 3 & 4 & 12.96 \\
\hline
\end{tabular}

\subsection{Análisis de ambos sistemas}

En esta sección se hizo un análisis de costos por sistema de techumbre, con el objeto de determinar la relación de Precio Unitario por Losa correspondiente. Los resultados se muestran en las Tablas 7 y 8

\subsection{Cost Analysis for Both Systems}

In this stage a cost analysis was made in order to determine the $U$ nit Price ratio per each roofing system. The results are shown in Tables 7 and 8.

Tabla 7. Análisis de costos unitarios para el sistema tradicional

Table 7. U nit Costs Analisys for the traditional system

\begin{tabular}{|c|c|c|c|c|c|c|}
\hline $\begin{array}{l}\text { Dato simulación } \\
\text { Simulation datum }\end{array}$ & $\begin{array}{l}\text { Duración (Tabla 5) } \\
\text { Duration (Table 5) }\end{array}$ & $\begin{array}{c}\text { Costo directo }(\mathrm{cd})(\text { tabla } 3) \\
\text { Direct cost }(\mathrm{dc})(\text { table } 3)\end{array}$ & $\begin{array}{c}\text { Costo indirecto de administración }(\mathrm{Cl}) \\
\text { Administration non-direct cost (N O N-DC) }\end{array}$ & $\begin{array}{l}\text { Total-Iosa } \\
\text { Roof-total }\end{array}$ & $\begin{array}{l}\text { Precio unitario total-losa } \\
\text { Unit price roof total }\end{array}$ & $\begin{array}{l}\text { Relación \% Cl/CD } \\
\% \text { Ratio DC/NON-DC }\end{array}$ \\
\hline $\mathrm{N}^{\circ}$ losas/roof NR. & Días/days & $\$ 8,435.88 \times \mathrm{A}$ & $\$ 507.27 \times A \times B$ & $C+D$ & $E / A$ & $D / C$ \\
\hline$A$ & B & C & D & $E$ & $\mathrm{~F}$ & G \\
\hline 1 & 2.73 & $\$ 8,435.88$ & $\$ 1,384.85$ & $\$ 9,820.73$ & $\$ 9,820.73$ & $16.42 \%$ \\
\hline 5 & 4.45 & $\$ 42,179.40$ & $\$ 11,286.76$ & $\$ 53,466.16$ & $\$ 10,693.23$ & $26.76 \%$ \\
\hline 10 & 4.89 & $\$ 84,358.80$ & $\$ 24,805.50$ & $\$ 109,164.30$ & $\$ 10,916.43$ & $29.40 \%$ \\
\hline 15 & 5.47 & $\$ 126,538.20$ & $\$ 41,621.50$ & $\$ 168,159.70$ & $\$ 11,210.65$ & $32.89 \%$ \\
\hline 20 & 6.4 & $\$ 168,717.60$ & $\$ 64,930.56$ & $\$ 233,648.16$ & $\$ 11,682.41$ & $38.48 \%$ \\
\hline 25 & 8.24 & $\$ 210,897.00$ & $\$ 104,497.62$ & $\$ 315,394.62$ & $\$ 12,615.78$ & $49.55 \%$ \\
\hline 30 & 8.1 & $\$ 253,076.40$ & $\$ 123,266.61$ & $\$ 376,343.01$ & $\$ 12,544.77$ & $48.71 \%$ \\
\hline 35 & 10.44 & $\$ 295,255.80$ & $\$ 185,356.46$ & $\$ 480,612.26$ & $\$ 13,731.78$ & $62.78 \%$ \\
\hline 40 & 10.06 & $\$ 337,435.20$ & $\$ 204,125.45$ & $\$ 541,560.65$ & $\$ 13,539.02$ & $60.49 \%$ \\
\hline 45 & 11.11 & $\$ 379,614.60$ & $\$ 253,609.64$ & $\$ 633,224.24$ & $\$ 14,071.65$ & $66.81 \%$ \\
\hline 50 & 12.09 & $\$ 421,794.00$ & $\$ 306,644.72$ & $\$ 728,438.72$ & $\$ 14,568.77$ & $72.70 \%$ \\
\hline 55 & 13.18 & $\$ 463,973.40$ & $\$ 367,720.02$ & $\$ 831,693.42$ & $\$ 15,121.70$ & $79.25 \%$ \\
\hline 60 & 14.95 & $\$ 506,152.80$ & $\$ 455,021.19$ & $\$ 961,173.99$ & $\$ 16,019.57$ & $89.90 \%$ \\
\hline 65 & 16.31 & $\$ 548,332.20$ & $\$ 537,782.29$ & $\$ 1,086,114.49$ & $\$ 16,709.45$ & $98.08 \%$ \\
\hline 70 & 16.94 & $\$ 590,511.60$ & $\$ 601,520.77$ & $\$ 1,192,032.37$ & $\$ 17,029.03$ & $101.86 \%$ \\
\hline 75 & 17.92 & $\$ 632,691.00$ & $\$ 681,770.88$ & $\$ 1,314,461.88$ & $\$ 17,526.16$ & $107.76 \%$ \\
\hline 80 & 18.87 & $\$ 674,870.40$ & $\$ 765,774.79$ & $\$ 1,440,645.19$ & $\$ 18,008.06$ & $113.47 \%$ \\
\hline 85 & 19.85 & $\$ 717,049.80$ & $\$ 855,891.31$ & $\$ 1,572,941.11$ & $\$ 18,505.19$ & $119.36 \%$ \\
\hline 90 & 20.91 & $\$ 759,229.20$ & $\$ 954,631.41$ & $\$ 1,713,860.61$ & $\$ 19,042.90$ & $125.74 \%$ \\
\hline 95 & 21.87 & $\$ 801,408.60$ & $\$ 1,053,929.52$ & $\$ 1,855,338.12$ & $\$ 19,529.87$ & $131.51 \%$ \\
\hline 100 & 21.87 & $\$ 843,588.00$ & $\$ 1,109,399.49$ & $\$ 1,952,987.49$ & $\$ 19,529.87$ & $131.51 \%$ \\
\hline
\end{tabular}


Tabla 8. Análisis de costos unitarios para el sistema L-18

Table 8. U nit Costs Analysis for the the precast L-18 roofing system

\begin{tabular}{|c|c|c|c|c|c|c|}
\hline $\begin{array}{l}\text { Dato simulación } \\
\text { Simulation datum }\end{array}$ & $\begin{array}{c}\text { Duración (Tabla 6) } \\
\text { Duration (Table 6) }\end{array}$ & $\begin{array}{l}\text { Costo directo }(\mathrm{cd})(\text { tabla } 4) \\
\text { Direct cost }(\mathrm{dc})(\text { table } 4)\end{array}$ & $\begin{array}{c}\text { Costo indirecto de administración }(\mathrm{Cl}) \\
\text { Administration non-direct cost (N ON-DC) }\end{array}$ & $\begin{array}{l}\text { Total-losa } \\
\text { Roof-total }\end{array}$ & $\begin{array}{c}\text { Precio unitario total-losa } \\
\text { Unit price roof total }\end{array}$ & $\begin{array}{l}\text { Relación \% Cl/CD } \\
\% \text { Ratio DC/NON-DC }\end{array}$ \\
\hline $\mathrm{N}^{\circ}$ losas/roof NR. & Días/days & $\$ 11,523.15 \times A$ & $\$ 507.27 \times A \times B$ & $C+D$ & $E / A$ & $D / C$ \\
\hline A & B & $C$ & D & $\mathrm{E}$ & $\mathrm{F}$ & G \\
\hline 1 & 1.8 & $\$ 11,575.35$ & $\$ 913.09$ & $\$ 12,436.24$ & $\$ 12,436.24$ & $7.92 \%$ \\
\hline 5 & 2.84 & $\$ 57,876.75$ & $\$ 7,203.23$ & $\$ 64,818.98$ & $\$ 12,963.80$ & $12.50 \%$ \\
\hline 10 & 3.83 & $\$ 115,753.50$ & $\$ 19,428.44$ & $\$ 134,659.94$ & $\$ 13,465.99$ & $16.86 \%$ \\
\hline 15 & 4.67 & $\$ 173,630.25$ & $\$ 35,534.26$ & $\$ 208,381.51$ & $\$ 13,892.10$ & $20.56 \%$ \\
\hline 20 & 5.84 & $\$ 231,507.00$ & $\$ 59,249.14$ & $\$ 289,712.14$ & $\$ 14,485.61$ & $25.71 \%$ \\
\hline 25 & 6.91 & $\$ 289,383.75$ & $\$ 87,630.89$ & $\$ 375,709.64$ & $\$ 15,028.39$ & $30.42 \%$ \\
\hline 30 & 8.01 & $\$ 347,260.50$ & $\$ 121,896.98$ & $\$ 467,591.48$ & $\$ 15,586.38$ & $35.26 \%$ \\
\hline 35 & 9.24 & $\$ 405,137.25$ & $\$ 164,051.12$ & $\$ 567,361.37$ & $\$ 16,210.32$ & $40.68 \%$ \\
\hline 40 & 10.44 & $\$ 463,014.00$ & $\$ 211,835.95$ & $\$ 672,761.95$ & $\$ 16,819.05$ & $45.96 \%$ \\
\hline 45 & 8.85 & $\$ 520,890.75$ & $\$ 202,020.28$ & $\$ 720,562.03$ & $\$ 16,012.49$ & $38.96 \%$ \\
\hline 50 & 9.72 & $\$ 578,767.50$ & $\$ 246,533.22$ & $\$ 822,690.72$ & $\$ 16,453.81$ & $42.79 \%$ \\
\hline 55 & 10.8 & $\$ 636,644.25$ & $\$ 301,318.38$ & $\$ 935,091.63$ & $\$ 17,001.67$ & $47.54 \%$ \\
\hline 60 & 11.2 & $\$ 694,521.00$ & $\$ 340,885.44$ & $\$ 1,032,274.44$ & $\$ 17,204.57$ & $49.30 \%$ \\
\hline 65 & 9.7 & $\$ 752,397.75$ & $\$ 319,833.74$ & $\$ 1,068,838.49$ & $\$ 16,443.67$ & $42.70 \%$ \\
\hline 70 & 10.25 & $\$ 810,274.50$ & $\$ 363,966.23$ & $\$ 1,170,586.73$ & $\$ 16,722.67$ & $45.12 \%$ \\
\hline 75 & 10.96 & $\$ 868,151.25$ & $\$ 416,975.94$ & $\$ 1,281,212.19$ & $\$ 17,082.83$ & $48.25 \%$ \\
\hline 80 & 11.62 & $\$ 926,028.00$ & $\$ 471,558.19$ & $\$ 1,393,410.19$ & $\$ 17,417.63$ & $51.15 \%$ \\
\hline 85 & 10.46 & $\$ 983,904.75$ & $\$ 451,013.76$ & $\$ 1,430,481.51$ & $\$ 16,829.19$ & $46.05 \%$ \\
\hline 90 & 11.71 & $\$ 1,041,781.50$ & $\$ 534,611.85$ & $\$ 1,571,695.35$ & $\$ 17,463.28$ & $51.55 \%$ \\
\hline 95 & 12.55 & $\$ 1,099,658.25$ & $\$ 604,792.66$ & $\$ 1,699,491.91$ & $\$ 17,889.39$ & $55.25 \%$ \\
\hline 100 & 12.96 & $\$ 1,157,535.00$ & $\$ 657,421.92$ & $\$ 1,809,736.92$ & $\$ 18,097.37$ & $57.05 \%$ \\
\hline
\end{tabular}

Combinando los datos de las columnas de Precios U nitarios Totales por Losa de las Tablas 7 y 8 y graficándolas contra el Número de Losas correspondiente, se obtuvo los resultados que se muestran en la Figura 5.
The results of combining data from column Total U nit Prices per Roof in Tables 7 and 8, versus the corresponding roofing number, are shown in Figure 5.

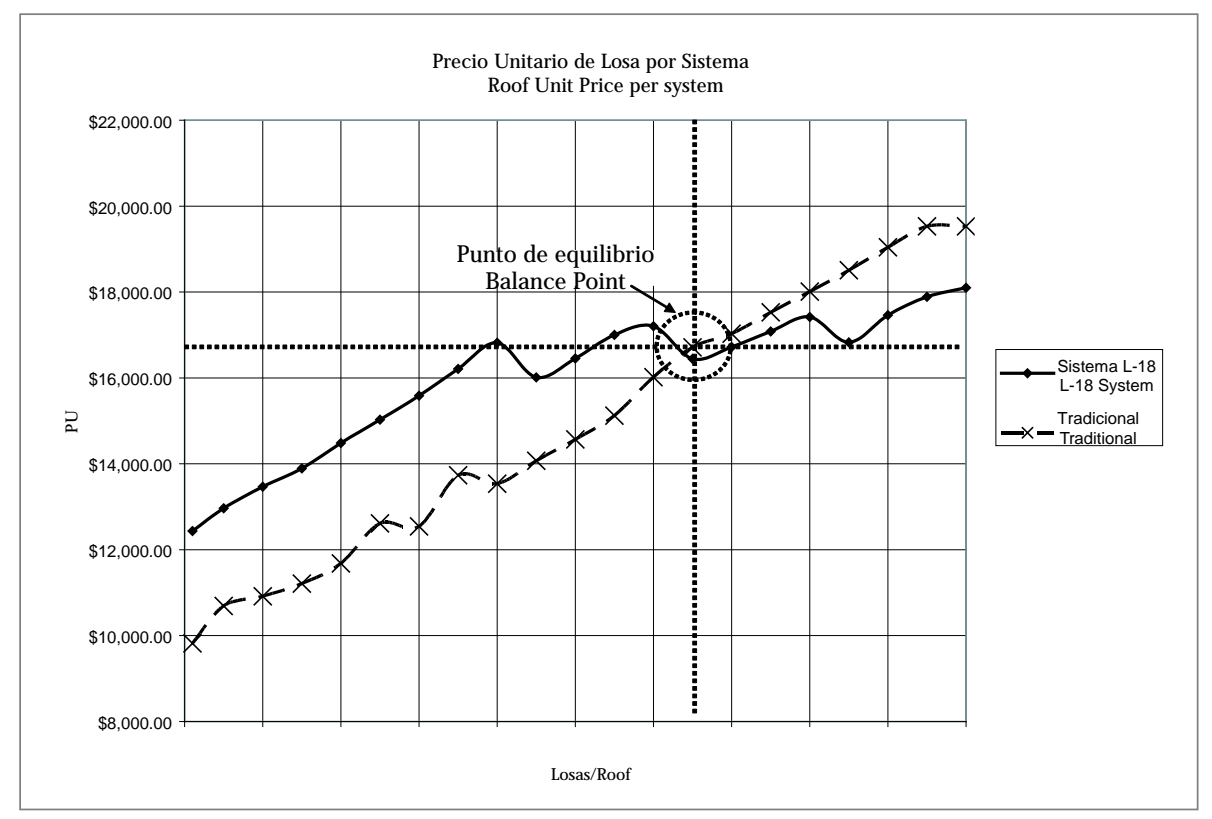

Figura 5. Precios U nitarios de construcción masiva de losas de viviendas, utilizando el sistema tradicional y el sistema prefabricado L-18

Figure 5. U nit Prices M assively Construction Housing Roofing System, using the traditional system and precast L-18system 
A partir de esta gráfica, se puede decir que se ha hallado el punto de equilibrio. En la Figura 5 se puede notar que el sistema L-18 empieza a hacerse más económico para la empresa, en cuestiones relacionadas al costo unitario, a partir de 65 losas.

Combinando los datos de la columna de Costo Indirecto sobre Costo Directo de las Tablas 7 y $8(\mathrm{Cl} /$ CD), y graficándolas contra el Número de Losas correspondiente, se obtuvo los resultados que se muestran en la Figura 6

En la Figura 6 se puede hacer notar que la relación porcentual del $\mathrm{Cl} / \mathrm{CD}$ se va manteniendo por debajo del $60 \%$ para el método prefabricado L-18. Por otra parte, este índice tiene una tendencia a incrementarse para el método tradicional de vigueta y bovedilla.
According to this graph, a point of balance seems to be found. Regarding unit cost, Figure 5 shows that the cost of the L-18 system becomes lower than traditional system when the company builds 65 roofing systems ot more.

The percentage ratio from Non-Direct and Direct Cost Columns in Tables 7 and 8 (DC/NON-DC), versus the corresponding roofing number is shown in Figure 6.

Figure 6 shows that the percentage relation DC/NON-DC keeps below $60 \%$ for precast L-18 system. Besides, this index tends to increase for traditional ceiling hoist and vault system.

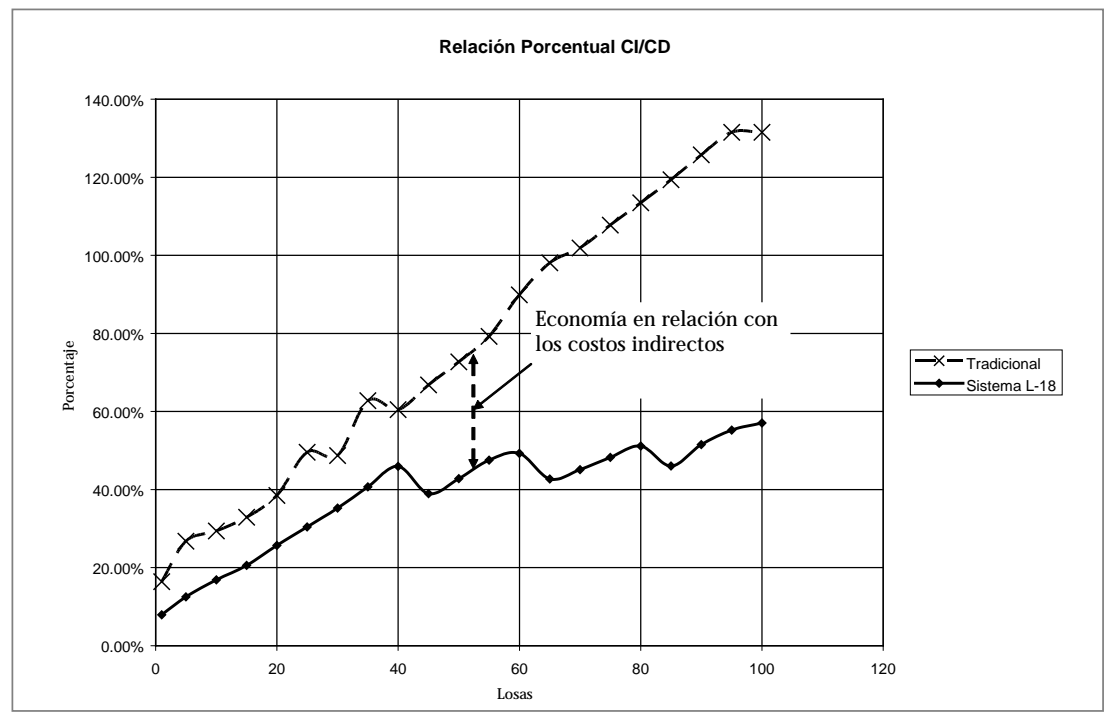

Figura 6. Relación porcentual entre los costos indirectos y directos ( $\mathrm{Cl} / \mathrm{CD})$ Figure 6. Percentage Ratio between Direct and Non-Direct Costs

\section{Discusión}

Los modelos propuestos pueden ser mejorados, pero representan el siguiente paso para comparar el sistema tradicional con otros que estén representados de la misma manera, tal como los estudiados por (Sosa et al., 2007). Se puede decir que los modelos de simulación masivos presentados en este trabajo difieren de los presentados por Baeza y por Sosa. Sin embargo los modelos presentados en este trabajo, tuvieron como punto de partida los mencionados en trabajos previos. Estos modelos ya toman en consideración la información sobre recursos y sobre costos indirectos.

\section{Discussion}

Proposed models can be improved, but they would become the next step for comparing traditional systems to others expressed in the same way as the ones studied by (Sosa et. al, 2007). However, models shown in this study were based on previous works. These models already take into consideration data related to resources and non-direct costs. 
Se puede mencionar que la información sobre recursos para ambos sistemas fue tomada de una entrevista con un residente de obra. Esto se puede mejorar teniendo mayor participación de más residentes para afinar y poner a punto la información para la simulación en ambos sistemas de techumbre.

Aunque se puede decir que el empleo de prefabricados en la construcción de losas para casas habitación es caro, se puede economizar si el volumen de obra es alto. Este trabajo se puede tomar como base para optimizar los procesos de las empresas constructoras de la región sureste de México. El punto de equilibrio entre los dos sistemas hallado en este trabajo, demuestra que los costos indirectos de la empresa repercuten sobre la ganancia monetaria que una empresa constructora tiene.

Aunque el sistema tradicional tiene la gran ventaja de ser más económico (compárense la columna E de las Tablas 7 y 8), esta economía es aparente para la empresa constructora (véase la Figura 6). La relación entre los costos indirectos e indirectos indica que la empresa puede llegar a pagar más en cuestiones de administración, que el costo directo de producción (cerca del 100\% para 65 losas).

\section{Conclusión}

El tiempo de fabricación de las losas para un proyecto de construcción de vivienda resultó ser más productivo con el sistema prefabricado que con el sistema de losa de vigueta y bovedilla en volúmenes masivos de obra. El precio unitario total de producción por losa para una empresa constructora durante la fabricación masiva de vivienda involucra la suma de dos costos unitarios parciales: el costo directo, que contempla los costos de mano de obra y materiales y costo indirecto por administración, que engloba a los costos referidos a sueldos de oficina y campo, las prestaciones por ley y los gastos de operación.

En lo que se refiere al costo directo se observó que el método prefabricado es mayor que el método de vigueta y bovedilla, sin embargo, el sistema prefabricado es capaz de ajustar y adaptar su precio de venta en la planta en función de un volumen determinado de obra, según se pudo ver en la Figura 5 dando como resultado la disminución de este porcentaje directo (Figura 6).
The information about resources for both systems was obtained from an interview with the building supervisor. However data can be improved by interviewing more workers in order to count with the exact and complete information for the simulation of two roofing systems.

Even though the use of precasting in the housing roofing units construction is quite expensive, savings can be made when the construction volume is high. This study can be taken into consideration, as a starting point to improve processes in construction companies at the southeast M exico region. The balance point found between two systems determines the financial profit a construction company obtains.

Although traditional system has the advantage of being more economic (compare column E in Tables 7 and 8 ), this saving is only apparent for the construction company (See Figure 6). The relation direct costs and non-direct costs indicates that the company could even pay more in administrative issues than the production direct cost (close to $100 \%$ for 65 roofing systems).

\section{Conclusion}

Execution period for roofing systems in a housing construction project, happened to be more productive when using the precast system than the ceiling joist and vault system for massively construction volumes. The total unit price per roofing system, for a construction company, during massively housing construction involves the addition of two partial unit costs: direct cost that includes labor hand and raw material, and non-direct administrative cost that includes office and field expenses, services payment and operational costs.

As far as direct cost is concerned, the precast system is higher than the ceiling joist and vault system, however, the precast system is well able to adjust and to adapt its selling price based on a given construction volume, as seen in Figure 5, thus resulting a decrease in the direct percentage (Figure 6). 


\section{Referencias / References}

Baeza Pereyra y Julio R. (2008), Modelo de simulación para fabricar losa tradicional en la región sureste de M éxico, Ingeniería y U niversidad, Pontificia U niversidad Javeriana, Vol. 12, num. 1

Campos Silvia (1985), Costo de una vivienda de interés social, monografía inédita, U niversidad Autónoma De Yucatán. Gutierrez Aída (1995), Defectos de procesos constructivos, monografía inédita, Universidad Autónoma De Yucatán. Martínez J. C. (1998), "EZStrobe - General-Purpose Simulation System Based on Activity Cycle, Diagrams", Proceedings of the 1998 W inter Simulation Conference held in Washington, D.C., December 13-16.

Pinzón José (1990), Estudio de rendimientos de mano de obra en la construcción de viviendas de interés social, tesis inédita, Universidad Autónoma De Yucatán.

Sosa Canto Jorge A., Baeza Pereyra Julio R. y Arcudia Abad Carlos E. (2007), Modelos para la simulación computarizada del proceso constructivo de losa prefabricada L-18, Ingeniería y U niversidad, Pontificia U niversidad Javeriana, Vol. 11, num. 1.

Sosa Canto Jorge A. (2005), Evaluación técnico-económica entre dos tipos de losa mediante un proceso de simulación, tesis inédita, Universidad Autónoma De Yucatán.

Yee Alfred ( 2001), Structural and economic benefits of precast/prestressed concrete construction, Pc Journal (IIlinois), 46:, núm 4. 